\title{
CONVOLUTION EQUATIONS IN SPACES OF INFINITE DIMENSIONAL ENTIRE FUNCTIONS OF EXPONENTIAL AND RELATED TYPES
}

BY

\author{
J. F. COLOMBEAU AND B. PERROT
}

\begin{abstract}
ABSTRaCr. We prove results of existence and approximation of the solutions of the convolution equations in spaces of entire functions of exponential type on infinite dimensional spaces. In particular we obtain: let $E$ be a complex, quasi-complete and dual nuclear locally convex space and $\Omega$ a convex balanced open subset of $E$; let $\mathcal{H C}(\Omega)$ be the space of the holomorphic functions on $\Omega$, equipped with the compact open topology and $\mathcal{H}^{\prime}(\Omega)$ its strong dual; let $\mathscr{F} \mathcal{H}^{\prime}(\Omega)$ denote the image of $\mathcal{K}^{\prime}(\Omega)$ through the Fourier-Borel transform $\mathscr{F}$; equip this space $\mathcal{F} \mathcal{X C}^{\prime}(\Omega)$ with the image of the topology of $\mathcal{K}^{\prime}(\Omega)$ via the map $\mathscr{F}$. Then, "every nonzero convolution operator on $\mathscr{F} \mathcal{H}^{\prime}(\Omega)$ is surjective" and "every solution of the homogeneous equation is limit of exponential-polynomial solutions". Our results are more generally valid when $E$ is a Schwartz bornological vector space with the approximation property. Previous results in Fréchet-Schwartz and Silva spaces are thus extended to domains that are not Fréchet or D.F.-spaces.
\end{abstract}

1. Introduction. Let $E$ be a complex Hausdorff locally convex space and $\Omega$ a convex balanced open subset of $E$; let $\mathcal{H}(\Omega)$ denote the space of the holomorphic functions on $\Omega$ [10] equipped with the compact open topology; let $\mathscr{F}$ denote the Fourier-Borel transform.

In some very usual cases for $E\left(E=\mathscr{D}\left(\mathbf{R}^{3} ; \mathbf{C}\right), \delta\left(\mathbf{R}^{3} ; \mathbf{C}\right), \ldots\right.$ using classical notations of L. Schwartz) the image, denoted by $\mathscr{F} \mathcal{H}^{\prime}(\Omega)$, of $\mathcal{H}^{\prime}(\Omega)$ through the Fourier Borel transform $\mathscr{F}$, is densely embedded in the Fock space of some Boson fields [5].

Functionals of annihilation operators motivate the study of the solvability of convolution equations in subspaces of $\mathscr{F} \mathcal{F C}^{\prime}(\Omega)$. Previous existence theorems, e.g. [8], [9], [10], have been restricted to the case when $E$ is a Fréchet space or a dual of a Fréchet space.

By means of a representation of $\mathscr{F} \mathcal{F C}^{\prime}(\Omega)$ as a bornological inductive limit of Silva spaces we are able to extend such results in this paper to nonmetrizable spaces such as $E=\mathscr{D}$ and $\mathscr{D})^{\prime}$ (distributions).

2. Notations and main definitions. In order to state the results in their natural setting and also for the convenience of some parts of the proofs we use the concept of the "convex bornological vector spaces" introduced in [16] and the concept of

Received by the editors April 30, 1979.

AMS (MOS) subject classifications (1970). Primary 46G20, 35R15; Secondary 32A10, $35 \mathrm{E} 99$.

Key words and phrases. Infinite-dimensional holomorphy, convolution equations, Fourier-Borel transform, bornology. 
the Silva holomorphic functions ([4]). A result of [7] asserts that, in the usual cases for $E$, the space of the holomorphic (i.e. $G$-analytic and continuous) functions on $\Omega$ is dense in the space of the Silva holomorphic functions on $\Omega$ for the compact open topology. Hence they have the same dual denoted by $\mathcal{H C}^{\prime}(\Omega)$. From now on, $\mathcal{H}(\Omega)$ will denote the space of the Silva holomorphic functions on $\Omega$ (to be defined below).

We recall ([16], [13], [14]) that a complete convex bornological vector space $E$ (denoted by the letters b.v.s.) is an injective algebraic inductive limit of a family $\left(E_{i}\right)_{i \in I}$ of Banach spaces such that if $i \leqslant j$, the canonical linear map from $E_{i}$ to $E_{j}$ is injective and continuous. A subset of $E$ is said to be bounded iff it is contained and bounded in a Banach space $E_{i}$. Two such structures are identified iff they have the same bounded sets.

We recall ([14]) that $E$ is a Schwartz b.v.s. iff for each index $i$ there exists an index $j \in I$ such that the unit ball $B_{i}$ of the Banach space $E_{i}$ is relatively compact in the Banach space $E_{j}$.

$\Omega$ denotes a convex balanced subset of $E$ such that, for each index $i, \Omega \cap E_{i}$ is open in the Banach space $E_{i}$. Let us recall that a subset $K$ of $\Omega$ is said to be strictly compact in $\Omega$ iff it is contained and compact in some open set $\Omega \cap E_{i}$ equipped with the topology induced by that of the Banach space $E_{i}$. In most of the usual locally convex spaces, the strictly compact sets coincide with the compact sets.

In this paper $E$ denotes a Schwartz b.v.s. separated by its dual and such that there exists a basis $\left(c_{i}\right)$ of convex balanced bounded sets such that the normed space $E_{c_{i}}$, spanned by $c_{i}$ and normed with the gauge of $c_{i}$, is a Banach space with the approximation property. These assumptions ensure the injectivity of the Fourier-Borel transform.

REMARK 1. Each nuclear b.v.s. $E$ admits a basis $\left(c_{i}\right)$ of convex balanced bounded sets such that each $E_{c_{i}}$ is a separable Hilbert space ([12], [13]). Hence the above assumption on $E$ is satisfied in a lot of usual spaces.

A Silva holomorphic function $f$ on $\Omega$ is a $G$-analytic function such that (if the b.v.s. $E$ is the injective algebraic inductive limit of the Banach spaces $E_{i}$ ) each restriction of $f$ to $\Omega \cap E_{i}$ is holomorphic (in the usual sense of Banach spaces) on the open subset $\Omega \cap E_{i}$ of the Banach space $E_{i}$. The space $\mathcal{H}(\Omega)$ (of the Silva holomorphic functions on $\Omega$ ) is equipped with the topology of the uniform convergence on the strictly compact subsets of $\Omega$.

REMARK 2. When $E$ is a dual nuclear l.c.s. the following description of $\mathscr{F} \mathcal{H C}^{\prime}(E)$ holds ([1], [2]). $\mathscr{F} \mathcal{H}^{\prime}(E)$ is the subspace of the homolorphic functions on the strong dual $E^{\prime}$ of $E$ such that there exists a convex balanced bounded subset $B$ of $E$ and a real number $c \geqslant 0$ such that

$$
|\phi(T)| \leqslant c e^{|T|_{B}}
$$

for each $T$ in $E^{\prime}$ (where $\left.|T|_{B}=\sup _{x \in B}|T(x)|\right)$.

Let us denote as usual by $E^{\times}$the bornological dual of the b.v.s. $E ; E^{\times}$is equipped with its natural bornology of the equibounded sets.

We recall that, in the general case, $\mathscr{F} \mathcal{H}^{\prime}(\Omega)$ is contained in $\mathcal{H}\left(E^{\times}\right)$and, for $\alpha$ in $E^{\times}$, we denote by $\tau_{\alpha}$ the translation operator, $\left(\tau_{\alpha} \phi\right)(\beta)=\phi(\alpha+\beta)$ where $\alpha, \beta$ are 
in $E^{\times}$and $\phi$ is in $\mathcal{H}\left(E^{\times}\right)$and it is proved (Lemma 1) that $\tau_{\alpha}$ is a linear continuous map from $\mathscr{F} \mathcal{H}^{\prime}(\Omega)$ to $\mathscr{F} \mathcal{H}^{\prime}(\Omega)$.

As usual, a linear continuous map from $\mathscr{F} \mathcal{F}^{\prime}(\Omega)$ to $\mathscr{F} \mathcal{H}^{\prime}(\Omega)$ which commutes with the translations is called a convolution operator on $\mathscr{F} \mathcal{F C}^{\prime}(\Omega)$.

3. Statement of the results. Let $E$ be a Schwartz b.v.s. separated by its dual and such that there exists a basis $\left(c_{i}\right)$ of convex balanced bounded sets such that each $E_{c_{1}}$ is a Banach space with the approximation property. For example, $E=\mathscr{D}(\Omega)$, $\mathscr{D}^{\prime}(\Omega), \mathcal{E}(\Omega), \mathcal{E}^{\prime}(\Omega), \mathcal{S}\left(\mathbf{R}^{n}\right), \mathcal{S}^{\prime}\left(\mathbf{R}^{n}\right), \mathcal{H}(\Omega), \mathcal{H}(K), \mathcal{H}^{\prime}(\Omega), \ldots$ with the usual notations for these spaces of finite-dimensional functions.

EXISTENCE THEOREM. Every nonzero convolution operator on $\mathscr{F} \mathcal{F C}^{\prime}(\Omega)$ is surjective.

Let $\theta$ be a convolution operator on $\mathscr{F} \mathcal{F C}^{\prime}(\Omega)$. Let $P \mathcal{E} x p \Omega$ denote the vector subspace of $\mathscr{F} \mathcal{H}^{\prime}(\Omega)$ spanned by the functions of the type $P e^{x}$ where $P$ is in $E^{\otimes_{n}}$ for some variable $n$ and where $x$ is in $\Omega$.

APPROXIMATION THEOREM. Every solution $u$ of the homogeneous equation $\theta u=0$ is a limit for the topology of $\mathscr{F} \mathcal{H C}^{\prime}(\Omega)$ of solutions in $P \mathcal{E} x p \Omega$.

\section{Proof.}

LEMMA 0. The strong topology $\mathcal{H}_{\beta}^{\prime}(\Omega)$ is the bornological topology of the equicontinuous bornology of $\mathcal{H}^{\prime}(\Omega)$.

Proof. From [7] $\mathcal{H}(\Omega)$ (Silva holomorphic functions) is a "completely reflexive l.c.s." according to the terminology of [14]. If $T \mathcal{F C}^{\prime}(\Omega)$ denotes the above bornological topology, the dual of $T \mathcal{H}^{\prime}(\Omega)$ is $\mathcal{H}(\Omega)$. Hence by Mackey's theorem [3] the strong topology on $\mathcal{H}^{\prime}(\Omega)$ is stronger than $T \mathcal{H}^{\prime}(\Omega)$. The converse is obvious.

LEMMA 1. For each $\alpha$ in $E^{\times}$the translation operator $\tau_{\alpha}$ is linear continuous from $\mathscr{F} \mathcal{H}^{\prime}(\Omega)$ to $\mathscr{F} \mathcal{H}^{\prime}(\Omega)$.

Proof. It suffices to show that it is linear bounded (Lemma 0). It is immediate to show that via the map $\mathcal{F}^{-1}$ (we recall that $\mathscr{F}$ is an injective map) $\tau_{\alpha}$ correspond to the map $l \rightarrow e^{\alpha} l$ from $\mathcal{H}^{\prime}(\Omega)$ to $\mathcal{H}^{\prime}(\Omega)$ (where $\left(e^{\alpha} l\right)(\varphi)=l\left(e^{\alpha} \cdot \varphi\right)$ if $l$ is in $\mathcal{H}^{\prime}(\Omega)$ and if $\varphi$ is in $\mathcal{H}(\Omega)$ ) and this last map is linear bounded from $\mathcal{H}^{\prime}(\Omega)$ to $\mathcal{H}^{\prime}(\Omega)$.

Let $T$ be in $\left(\mathscr{F} \mathcal{F C}^{\prime}(\Omega)\right)^{\prime}$ and let $T *$ be the map from $\mathscr{F} \mathcal{F C}^{\prime}(\Omega)$ to $\mathcal{H}\left(E^{\times}\right)$defined by $(T * \phi)(\alpha)=T\left(\tau_{\alpha} \phi\right)$ (it is easy to prove that the range of this map is in $\mathcal{H}\left(E^{\times}\right)$).

LEMMA 2. $T *$ is a convolution operator on $\mathscr{F}_{\mathcal{H}}(\Omega)$ and conversely each convolution operator on $\mathscr{F} \mathcal{H}^{\prime}(\Omega)$ is a $T *$ for some $T$. If $\mathfrak{U}$ denotes the algebra of the convolution operators on $\mathscr{F} \mathcal{H}^{\prime}(\Omega)$, the map

is a bijection.

$$
\begin{array}{ccc}
\left(\mathscr{F} \mathcal{F C}^{\prime}(\Omega)\right)^{\prime} & \rightarrow & \mathfrak{A} \\
T & \rightarrow & T *
\end{array}
$$

Proof. $\left(\mathscr{F} \mathcal{H}^{\prime}(\Omega)\right)^{\prime} \stackrel{t \mathscr{F}}{\rightarrow} \mathcal{H}$ " $(\Omega)=\mathcal{H}(\Omega)$ algebraically (since $\mathcal{H}(\Omega)$ is "completely reflexive" [7] it is a semireflexive 1.c.s.). If $T$ is in $\left(\mathscr{F} \mathcal{F}{ }^{\prime}(\Omega)\right)^{\prime}$ then ${ }^{t} \mathscr{F}(T)$ is an element of $\mathcal{H}(\Omega)$. The following diagram is commutative. 
where $U$ is the map defined by

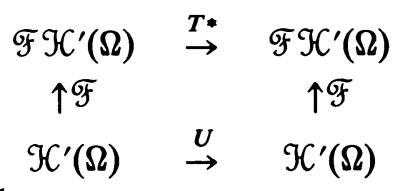

$$
\left.(U(l))(\psi)=l^{t} \mathscr{F}(T) \psi\right) \quad\left(l \in \mathcal{H}^{\prime}(\Omega), \psi \in \mathcal{H}(\Omega)\right)
$$

It is easy to prove that $U$ is bounded for the equicontinuous bornology on $\mathcal{H}^{\prime}(\Omega)$ hence continuous (Lemma 0 ). Hence $T *$ is continuous by definition of the topology of $\mathscr{F} \mathcal{F C}^{\prime}(\Omega)$.

Let $\gamma$ be the map

$$
\begin{aligned}
& \mathfrak{U} \rightarrow\left(\mathscr{F} \mathcal{H C}^{\prime}(\Omega)\right)^{\prime} \\
& \vartheta \rightarrow(\phi \rightarrow(\Theta \phi)(0)) \quad\left(\phi \in \mathscr{F F}^{\prime}(\Omega)\right),
\end{aligned}
$$

then $\gamma(T *)=T$ and $(\gamma \theta) *=\theta$.

$$
\mathscr{F}: \mathcal{H}^{\prime}(\Omega) \rightarrow \mathscr{F} \mathcal{H}^{\prime}(\Omega) \text { is an isomorphism hence }
$$

t $\mathscr{F}:\left(\mathscr{F} \mathcal{H C}^{\prime}(\Omega)\right)^{\prime} \rightarrow \mathcal{H}^{\prime \prime}(\Omega)=\mathcal{H}(\Omega)$ is a bijection.

LEMMA 3. If $S$ is in $\left(\mathscr{F} \mathcal{H}^{\prime}(\Omega)\right)^{\prime}$ then ${ }^{t} \mathscr{F}(S)$ is the element of $\mathcal{H}(\Omega)$ defined by $\left({ }^{t} \mathscr{F}(S)\right)(x)=S\left(e^{x}\right)$.

The proof is immediate if we recall that $\mathcal{H}^{\prime \prime}(\Omega)$ is identified with $\mathcal{H}(\Omega)$ by the formula $\phi\left(\delta_{x}\right)=\phi(x)$ (where $\delta_{x} \in \mathcal{H}^{\prime}(\Omega)$ is the Dirac measure at the point $x$ ).

Let us recall that $\mathscr{E x p}(\Omega) \subset \mathcal{H}\left(E^{\times}\right)$is the vector span of the functions $e^{x}$ where $x$ is in $\Omega$.

LEMMA 4. $\mathcal{E} x p \Omega$ is dense in $\mathscr{F} \mathcal{H}^{\prime}(\Omega)$.

Proof. Let $S$ be an element of $\left(\mathscr{F} \mathcal{F C}^{\prime}(\Omega)\right)^{\prime}$ such that $S\left(e^{x}\right)=0$ for each $x$ in $\Omega$. By Lemma 3, $t \mathscr{F}(S)=0$ in $\mathcal{H}(\Omega)$ hence $S=0$ in $\left(\mathscr{F} \mathcal{F} \mathcal{C}^{\prime}(\Omega)\right)^{\prime}$.

If $X, Y$ are in $\left(\mathscr{F} \mathcal{F C}^{\prime}(\Omega)\right)^{\prime}$ we define an element $X * Y$ of $\left(\mathscr{F} \mathcal{H}^{\prime}(\Omega)\right)^{\prime}$ by the formula

$$
t \mathscr{F}(X * Y)={ }^{t} \mathscr{F}(X) \cdot t \mathscr{F}(Y) \in \mathcal{H}(\Omega),
$$

and the following two formulas are immediate from the definitions.

(1) $\forall x \in \Omega \quad(X * Y)\left(e^{x}\right)=X\left(e^{x}\right) \cdot Y\left(e^{x}\right)$.

(2) $\forall S, T \in\left(\mathscr{F} \mathcal{H}^{\prime}(\Omega)\right)^{\prime}$ then $\left({ }^{t}(T *)\right)(S)=T * S$.

LEMMA 5. Let $f, g$ in $\mathcal{H}(\Omega), g \neq 0$, be such that for each affine subspace $S$ of dimension 1 of $E$ in which $g$ is not identically zero, then the restriction $f_{/ s}$ is divisible by the restriction $g_{/ s}$, with the quotient as a holomorphic function in $S$. Then $f$ is divisible by $g$ with the quotient as a holomorphic function in $\Omega$.

Proof. Apply Proposition 13 of [11], which asserts the same result in the case of Banach spaces.

LemMA 6. Let $X, T$ be in $\left(\mathcal{F} \mathcal{F}^{\prime}(\Omega)\right)^{\prime}, T \neq 0$. Let us assume that if $P$ is in $E^{\otimes n}$ and if $x$ is in $\Omega$,

$$
T * P e^{x}=0 \Rightarrow X\left(P e^{x}\right)=0
$$

then ${ }^{t} \mathscr{F}(X)$ is divisible by ${ }^{t} \mathscr{F}(T)$ with the quotient as a holomorphic function in $\Omega$. 
Proof. Apply Lemma 5 and for more details see the proof of Proposition 14 of [11].

Proof of the Approximation Theorem. If $\theta=0$ the result is true since $P \mathcal{E} x p \Omega$ is dense in $\mathscr{F} \mathcal{H}^{\prime}(\Omega)$. If $\theta \neq 0$ let $T=\gamma \theta$ (hence $T *=\theta$ ). Let $X$ be in $\left(\mathscr{F} \mathcal{H}^{\prime}(\Omega)\right)^{\prime}$ and such that $X$ is null on $P \mathcal{E} x p \Omega \cap \operatorname{Ker} \theta: T * P e^{x}=0 \Rightarrow X\left(P e^{x}\right)=0$, hence by Lemma 6 there exists an element $h$ of $\mathcal{H}(\Omega)$ such that ${ }^{t} \mathscr{F}(X)=h^{t} \mathscr{F}(T)$. Let $\mathcal{Q}={ }^{t} \mathscr{F}^{-1}(h) \in\left(\mathcal{F}_{\mathcal{F}}(\Omega)\right)^{\prime}$. Then ${ }^{t} \mathscr{F}(\mathcal{Q})=h$ hence ${ }^{t} \mathscr{F}(X)={ }^{t} \mathscr{F} \mathcal{Q} \cdot{ }^{t} \mathscr{F} T$ ${ }^{t} \mathscr{F}(\mathcal{Q} * T)$ hence $X=\mathscr{2} * T=T * \mathscr{L}$ (by (1)). Hence (by (2)) $X={ }^{t}(T *)(\mathcal{Q})$ $={ }^{t} \vartheta(\mathcal{2})$. If $\phi$ is in $\operatorname{Ker} \theta, X(\phi)=\mathcal{2}(\vartheta \phi)=0$ hence the result.

LEMMA 7. Let $\theta$ be a nonzero convolution operator on $\mathscr{F} \mathcal{H C}^{\prime}(\Omega)$. Then ${ }^{t} \mathcal{O}\left(\mathcal{F} \mathcal{H C}^{\prime}(\Omega)\right)^{\prime}=(\operatorname{Ker} \theta)^{0}$, the polar of $\operatorname{Ker} \theta$ in $\left(\mathcal{F} \mathcal{F C}^{\prime}(\Omega)\right)^{\prime}$.

Proof. The inclusion ${ }^{t} \theta\left(\mathscr{F} \mathcal{F C}^{\prime}(\Omega)\right)^{\prime} \subset(\operatorname{Ker} \theta)^{0}$ is immediate. Conversely let $X$ be in $(\operatorname{Ker} \theta)^{0}$. Using the proof of the Approximation Theorem, $X={ }^{t} \theta(2)$ for some 2 in $\left(\mathscr{F} \mathcal{H}^{\prime}(\Omega)\right)^{\prime}$.

Lemma 8 (Surjectivity lemma In Silva SPaCes). Let $E$ and $F$ be two Silva spaces and $u$ a linear continuous map from $E$ to $F$ and let $t_{u}$ be its transpose: Then $u$ is surjective iff $t_{u}$ is injective and $t_{u}\left(F^{\prime}\right)$ is weakly closed in $E^{\prime}$.

This Lemma 8 is considered as a "well-known" result but we prefer to sketch its proof.

Proof. By Proposition 5, §4, Chapter IV of [3], $u$ is surjective iff $t_{u}$ is a topological isomorphism between $F^{\prime}$ and $t_{u}\left(F^{\prime}\right)$ for the weak topologies $\sigma\left(F^{\prime}, F\right)$ and $\sigma\left(t_{u}\left(F^{\prime}\right), E\right)$. This isomorphism follows from the closed graph theorem between the two Fréchet spaces $E^{\prime}$ and $t_{u}\left(F^{\prime}\right)$ and from the semireflexivity of $E$ and $F$.

LEMMA 9. Let $E$ be a Silva space. Then each nonzero convolution operator on $\mathscr{F} \mathcal{H}^{\prime}(\Omega)$ is surjective.

Proof. In this case ([6], [7]) $\mathcal{H}(\Omega)$ is a Fréchet-Schwartz space, hence $\mathcal{F} \mathcal{F C}^{\prime}(\Omega)$ is a Silva space and ${ }^{\prime} \theta$ is injective. Let $X$ be in $(\mathcal{F} \mathcal{F}(\Omega))^{\prime}$ and such that ${ }^{\prime} \theta(X)=0$. Let $T=\gamma \theta$, hence $T *=\theta$ and $T * X=0$ (by (2)). Hence ${ }^{t} \mathscr{F}(T * X)=0$ hence ${ }^{t} \mathscr{F}(T)^{t} \mathscr{F}(X)=0$ hence ${ }^{t} \mathscr{F}(X)=0$ hence $X=0$. It suffices now to apply Lemmas 7 and 8.

Let $\left(c_{n}\right)$ be an increasing sequence of convex balanced bounded subsets of $E$ such that each $c_{n}$ is relatively compact in the Banach space $E_{c_{n+1}}$ and that $E_{c_{n+1}}$ is a Banach space with the approximation property. Let $\mathcal{E}$ be the Silva space which is the bornological inductive limit of the spaces $E_{c_{n}}$. Consider $\Omega \cap \mathcal{E}$ as contained in the b.v.s. $\mathcal{E}$ and denote by $\mathcal{H}(\Omega \cap \mathcal{E})$ the space of the holomorphic functions on $\Omega \cap \mathcal{E}$ for the bornological structure of $\mathcal{E}$. Let $r$ denote the restriction map $r$ : $\mathcal{H}(\Omega) \rightarrow \mathcal{H}(\Omega \cap \mathcal{E})$.

LEMMA 10. $r(\mathcal{H}(\Omega))$ is dense in $\mathcal{H}(\Omega \cap \mathcal{E})$ for the topology of uniform convergence on the compact subsets of $\Omega \cap \mathcal{E}$.

The proof follows immediately from Lemma 11 below. 
LEMMA 11. If $E_{c}$ has the approximation property then the restriction map $\mathcal{H}(\Omega) \rightarrow$ $\mathcal{H}\left(\Omega \cap E_{c}\right)$ has a dense range.

Proof. If $f$ is in $\mathcal{H}\left(\Omega \cap E_{c}\right)$, approximate $f$ by its Taylor expansion at the origin. Since $E_{c}$ has the approximation property, each polynomial on $E_{c}$ can be approximated by finite linear combinations of finite products of continuous linear forms, and a continuous linear form on $E_{c}$ can be uniformly approximated on compact subsets of $E_{c}$ by restrictions of continuous linear forms on $E$.

Proof of The Existence Theorem. Let $\theta$ be a nonzero convolution operator on $\mathscr{F} \mathcal{H C}^{\prime}(\Omega)$. Consider the following commutative diagram

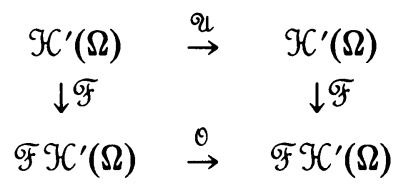

where $\mathscr{U}$ is the map induced by $\theta$ through the isomorphism $\mathscr{F}$. Obviously $\theta$ is surjective iff $\mathcal{Q}$ is surjective. If $K$ is a strictly compact subset of $E$ contained in $\Omega$ and if $\varepsilon$ is a strictly positive real number let

$$
V_{K, \varepsilon}=\left\{\varphi \in \mathcal{H}(\Omega) \text { such that } \sup _{x \in K}|\varphi(x)| \leqslant \varepsilon\right\} .
$$

Let $\stackrel{\circ}{K, \ell}_{K,}$ be its polar in $\mathcal{H}^{\prime}(\Omega)$. If $K$ is a strictly compact subset of $E$ contained in $\Omega$ then the closed convex balanced hull of $K$ is still contained in $\Omega$ (because $K$ is compact in some Banach space $E_{B}$ and the closed convex balanced hull of $K$ in $E_{B}$ is still contained in $\Omega$ ).

Let $y$ be an element of $H^{\prime}(\Omega)$. We shall show that there exists an element $x$ of $\mathcal{H}^{\prime}(\Omega)$ such that $\mathscr{U} x=y$. Since $\mathscr{U}$ is nonzero, let $z$ be an element of $\mathcal{H}^{\prime}(\Omega)$ such that $Q z \neq 0$.

The set $\{y, z\}$ is contained in some ${\stackrel{\circ}{K_{1}, e_{1}}}$ (where $K_{1}$ may be supposed convex balanced as already explained). In Lemma 2 it has been proved that $\mathscr{U}$ is bounded for the equicontinuous bornology on $\mathcal{H}^{\prime}(\Omega)$. Hence $\mathcal{Q}\left(\stackrel{\circ}{V}_{K_{1}, e_{1}}\right)$ is an equicontinuous set hence there exist $\varepsilon_{2}>0$ and a strictly compact subset $K_{2}^{\prime}$ contained in $\Omega$ such that $\mathcal{Q}\left({\stackrel{\circ}{K_{1}, \varepsilon_{1}}}\right) \subset{\stackrel{\circ}{K_{K^{\prime}, \varepsilon_{2}}}}$. Let $T_{1}$ be a convex and balanced strictly compact subset of $E$ such that $E_{K_{1}} \rightarrow E_{T_{1}}$ is compact. $\Omega \cap E_{K_{1}}$ is an open set in the Banach space $E_{K_{1}}$. Let $d$ (resp. $t$ ) be the distance in $E_{K_{1}}$ (resp. $E_{T_{1}}$ ) deduced from the norm of $E_{K_{1}}$ (resp. $E_{T_{1}}$ ). Let $K_{1}^{p}=\left\{x \in \Omega \cap E_{K_{1}}\right.$ such that $t\left(x, C_{E_{T_{1}}} \Omega\right) \geqslant 1 / p$ and $\left.d(x, 0)<p\right\}$.

Each compact set in $E_{K_{1}}$ which is contained in $\Omega$ is also compact in $E_{T_{1}}$ so it is

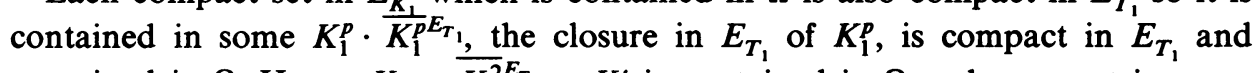
contained in $\Omega$. Hence $K_{1} \cup \bar{K}_{1}^{2} E_{T_{1}} \cup K_{2}^{\prime}$ is contained in $\Omega$ and compact in some Banach space $E_{c_{1}}$ where $c_{1}$ is a convex and balanced bounded subset of $E$. Hence the closed convex balanced hull of $K_{1} \cup K_{1}^{2} \cup K_{2}^{\prime}$ in $E_{c_{1}}$, denoted by $\bar{\Gamma}\left(K_{1} \cup K_{1}^{2} \cup\right.$ $\left.K_{2}^{\prime}\right)$, is still contained in $\Omega$. From the assumptions on the space $E$ one may assume that $E_{c_{1}}$ is a Banach space with the approximation property and that $c_{1}$ is strictly compact in $E$. Hence there exists $\alpha_{1}>0$ such that $\bar{\Gamma}\left(K_{1} \cup K_{1}^{2} \cup K_{2}^{\prime}\right)+\alpha_{1} c_{1} \subset \Omega$. Let $K_{2}=\bar{\Gamma}\left(K_{1} \cup K_{1}^{2} \cup K_{2}^{\prime}\right)+\alpha_{1} c_{1}$. Then $K_{2}$ is a convex balanced strictly compact subset of $E$ and $K_{2}$ is contained in $\Omega$. Define the sequence of subsets $\left(K_{2}^{p}\right)_{p \in N}$ in 
$\Omega \cap E_{K_{2}}$ in the same way as it was done before with $K_{1}$ instead of $K_{2}$. Hence each compact set in $E_{K_{2}}$ which is contained in $\Omega$ is contained in some $K_{2}^{p}$. There exists a strictly compact subset $K_{3}^{\prime}$ of $E, K_{3}^{\prime}$ contained in $\Omega$, such that $\mathscr{Q}\left(\dot{V}_{K_{2}, \ell_{2}}\right) \subset{\stackrel{\circ}{K_{3}^{\prime}, \ell_{3}}}$ $\left(\varepsilon_{3}>0\right)$ because we have already proved that the map $\mathscr{U}$ is bounded for the equicontinuous bornology of $\mathcal{H}^{\prime}(\Omega)$.

Now we shall do with $K_{2} \cup K_{1}^{3} \cup K_{2}^{3} \cup K_{3}^{\prime}$ the work already done with $K_{1} \cup K_{1}^{2}$ $\cup K_{2}^{\prime}: \bar{\Gamma}\left(K_{2} \cup K_{1}^{3} \cup K_{2}^{3} \cup K_{3}^{\prime}\right)$ is compact in some Banach space $E_{c_{2}}$ (with the approximation property) and contained in $\Omega$. Hence there exists $\alpha_{2}>0$ such that $\bar{\Gamma}\left(K_{2} \cup K_{1}^{3} \cup K_{2}^{3} \cup K_{3}^{\prime}\right)+\alpha_{2} c_{2} \subset \Omega$. Let $K_{3}=\bar{\Gamma}\left(K_{2} \cup K_{1}^{3} \cup K_{2}^{3} \cup K_{3}^{\prime}\right)+\alpha_{2} c_{2}$. Then $K_{3}$ is a convex balanced strictly compact subset of $E$ and is contained in $\Omega$. Define now the sequence $\left(K_{3}^{p}\right)_{p \in N}$ and $K_{4}^{\prime}$ such that $\mathscr{U}\left(\dot{V}_{K_{3}, e_{3}}\right) \subset \dot{V}_{K_{4, e_{4}}^{\prime}}$ The recurrence is obvious. We obtain sequences $K_{n}, K_{n}^{\prime}, c_{n}, \varepsilon_{n}>0, \alpha_{n}>0$ such that:

$$
K_{n+1}=\bar{\Gamma}\left(K_{n} \cup K_{1}^{n+1} \cup K_{2}^{n+1} \cup \cdots \cup K_{n}^{n+1} \cup K_{n+1}^{\prime}\right)+\alpha_{n} c_{n} \subset \Omega
$$

and $E_{K_{n}} \subset E_{c_{n}} \subset E_{K_{n+1}}$ with continuous inclusions.

Let $\mathscr{E}$ denote the inductive limit of the Banach spaces $E_{c_{n}}$ which is also the inductive limit of the Banach spaces $E_{K_{n}} \cdot \mathcal{E}$ is a Silva space and we may apply Lemma 10. The restriction map $r: \mathcal{H}(\Omega) \rightarrow \mathcal{H}(\Omega \cap \mathcal{E})$ has a dense range hence its transpose ${ }^{t} r: \mathcal{H}^{\prime}(\Omega \cap \mathcal{E}) \rightarrow \mathcal{H}^{\prime}(\Omega)$ is injective, hence via ${ }^{t} r, \mathcal{H}$ ' $(\Omega \cap \mathcal{E})$ may be identified as a subspace of $\mathcal{H}^{\prime}(\Omega)$. One may choose the sequence $\left(\varepsilon_{n}\right)$ converging to 0 hence since each compact set of $\mathcal{E}$ contained in $\Omega$ is contained in some $K_{n}$ (this follows from the construction of the sets $K_{n}$ via the sequences $\left.\left(K_{n}^{p}\right)_{p \in N}\right)$ the sets

$$
W_{K_{n}, e_{n}}=\left\{\varphi \in \mathcal{H}(\Omega \cap \mathcal{E}) \text { such that } \sup _{x \in K_{n}}|\varphi(x)|<\varepsilon_{n}\right\}
$$

are a basis of neighbourhoods of 0 in the compact open topology of $\mathcal{H}(\Omega \cap \mathcal{E})$. Hence their polars $\dot{W}_{K_{n}, e_{n}}$ in $\mathcal{K}^{\prime}(\Omega \cap \mathcal{E})$ are a basis of the equicontinuous bornology of $\mathcal{H}^{\prime}(\Omega \cap \mathcal{E})$.

From the density result obtained in Lemma 10 it follows easily that ${ }^{t} r\left(\dot{W}_{K_{n}, e_{n}}\right)=$ $\stackrel{\circ}{V}_{K_{n}, e_{n}}$. Since $\mathcal{Q}\left({\stackrel{\circ}{V_{K}, e_{n}}}\right) \subset{\stackrel{\circ}{K_{n+1}, e_{n+1}}}$, the subspace $\mathcal{H}^{\prime}(\Omega \cap \mathcal{E})$ is mapped into itself by $\mathcal{Q}$ and the restriction $\mathcal{Q}_{/ \mathcal{F}^{\prime}(\Omega \cap \mathcal{E})}$ of $\mathcal{U}$ to $\mathcal{H}^{\prime}(\Omega \cap \mathcal{E})$ is bounded for the equicontinuous bornology on $\mathcal{H}^{\prime}(\Omega \cap \mathcal{E})$.

Now we are going to show that $\mathcal{U}_{/ \mathcal{F}^{\prime}(\Omega \cap \mathcal{E})}$ induces a nonzero convolution operator on $\mathscr{F} \mathcal{H}^{\prime}(\Omega \cap \mathcal{E}$ ) (via the map $\mathscr{F}$ ). It is easy to show that the operator on $\mathscr{F}_{\mathcal{H}}(\Omega \cap \mathcal{E})$ induced by $\mathcal{U}_{/ \mathcal{F}^{\prime}(\Omega \cap \mathcal{E})}$ via the Fourier-Borel transform $\mathscr{F}$ is the restriction of $\theta$ to $\mathscr{F} \mathcal{H}^{\prime}(\Omega \cap \mathcal{E})$ (since $\mathcal{H}^{\prime}(\Omega \cap \mathcal{E})$ is considered as a subspace of $\mathcal{H}^{\prime}(\Omega), \mathscr{F}_{\mathcal{H}}(\Omega \cap \mathcal{E})$ is considered as a subspace of $\left.\mathscr{F} \mathcal{H}^{\prime}(\Omega)\right)$. The continuity of $\mathcal{O}_{/ \mathscr{F} \mathcal{F}^{\prime}(\Omega \cap \mathcal{E})}$ follows from the fact that $\mathcal{W}_{/ \mathcal{F C}^{\prime}(\Omega \cap \mathcal{E})}$ is bounded for the equicontinuous bornology on $\mathcal{H}^{\prime}(\Omega \cap \mathcal{E})$, that $\mathscr{F}$ is an isomorphism and from Lemma 0 . Since $z$ is in $\mathcal{H}^{\prime}(\Omega \cap \mathcal{E}), \theta_{/ \mathscr{F}} \mathcal{C}^{\prime}(\Omega \cap \mathcal{E})$ is nonzero. The fact that $\theta_{/ \mathscr{F} \mathcal{C}(\Omega \cap \mathcal{E})}$ is translation invariant for the translations $\tau_{\alpha}$ (where $\alpha$ is in $\mathcal{E}^{\times}$) comes easily from an argument of continuity and the fact that if $i$ denotes the natural injection from $\mathcal{E}$ to $E$ then its transpose ${ }^{t} i$ from $E^{\times}$to $\mathcal{E}^{\times}$has a dense range (because the Silva space $\mathcal{E}$ is semireflexive and because the b.v.s. $E$ is separated by its dual).

From Lemma 9 it follows that the convolution operator $\mathcal{\theta}_{/ \mathscr{F}^{\prime} \mathcal{C}^{\prime}(\Omega \cap \text { () }}$ is surjective. 
Since $y$ is in $\mathcal{H}^{\prime}(\Omega \cap \mathcal{E})$ there exists an element $x$ of $\mathcal{H}^{\prime}(\Omega \cap \mathcal{E})$ such that

$$
\mathrm{U}_{/ \mathcal{X C}^{\prime}(\Omega \cap \mathcal{E})}(x)=y \text {. }
$$

Considering $x$ in $\mathcal{H}^{\prime}(\Omega)$ we have $\mathscr{U} x=y$ hence the theorem is proved.

ACKNOWLEDGEMENTS. The authors thank P. J. Boland with whom they discussed these results and the referee for many helpful suggestions.

\section{REFERENCES}

1. P. J. Boland, Malgrange theorem for entire functions on nuclear spaces, Proceedings on Infinite Dimensional Holomorphy, Lecture Notes in Math., vol. 364, Springer-Verlag, Berlin and New York, 1974, pp. 135-144.

2. , Holomorphic functions on nuclear spaces, Trans. Amer. Math. Soc. 209 (1975), 275-281.

3. N. Bourbaki, Espaces vectoriels topologiques, Hermann, Paris, 1953, 1955.

4. J. F. Colombeau, On some various notions of infinite dimensional holomorphy, Proceedings on Infinite Dimensional Holomorphy, Lecture Notes in Math., vol. 364, Springer-Verlag, Berlin and New York, 1974, pp. 145-149.

5. J. F. Colombeau and B. Perrot, Infinite dimensional holomorphic normal forms of operators, Advances in Holomorphy (editor, J. A. Barroso), North-Holland Math Studies, no. 34, North-Holland, Amsterdam, 1979, pp. 249-274.

6. __ Transformation de Fourier-Borel et noyaux en dimension infinie, C. R. Acad. Sci. Paris Ser. A 284 (1977), 963-966.

7. J. F. Colombeau, R. Meise and B. Perrot, A density result in spaces of Silva holomorphic mappings, Pacific J. Math. (to appear).

8. T. A. W. Dwyer III, Equations différentielles d'ordre infinite dans des espaces localement convexes,

C. R. Acad. Sci. Paris Ser. A 281 (1975), 163-166.

9. , Differential operators of infinite order on locally convex spaces. I, Rend. Mat. (1) 10 (1977), 149-179.

10. __ Differential operators of infinite order on locally convex spaces. II, Rend. Mat. 10 (1978), 273-293.

11. C. Gupta, Malgrange theorem for nuclearly entire functions of bounded type on a Banach space, Nederl. Akad. Wetensch, Proc. Ser. A 73 = Indag. Math. 32 (1970), 356-358.

12. H. Hogbé-Nlend, Completion, tenseurs et nucléarité en bornologie, J. Math. Pures Appl. 49 (1970), 193-288.

13. __ Théorie des bornologies et applications, Lecture Notes in Math., vol. 213, Springer-Verlag, Berlin and New York, 1971.

14. Bornologies and functional analysis, North-Holland Math. Studies, no. 26, North-Holland, Amsterdam, 1977.

15. L. Nachbin, A glimpse at infinite dimensional holomorphy, Proceedings on Infinite Dimensional Holomorphy, Lecture Notes in Math., vol. 364, Springer-Verlag, Berlin and New York, 1974, pp. 69-79. 16. L. Waelbroeck, Some theorems about bounded structures, J. Funct. Anal. 1 (1967), 392-408.

U. E. R. de Mathématiques et d'Informatique, Université de Bordeaux I, 351, Cours de la Libération, 33405 Talence, France 